\title{
Multi-parameters Measurement Based on STM32 ARM of Dual-excited Electromagnetic Flow Meter in Partially Filled Pipes
}

Suo Ji

Department of Electronic Information Engineering

Suzhou Vocational University

Suzhou ,Jiangsu Province, China suoji1996@163.com

\author{
Qi Chunqing \\ Department of Electronic Information Engineering \\ Suzhou Vocational University \\ Suzhou ,Jiangsu Province, China \\ qichunqing@163.com
}

\author{
Zhang Hongbing \\ Department of Electronic Information Engineering \\ Suzhou Vocational University \\ Suzhou ,Jiangsu Province, China \\ zhb@jssvc.edu.cn
}

\begin{abstract}
The dual-excited electromagnetic flowmeter with arc-electrodes in the paper can measures liquid level and flow velocity. A pair of electrodes is set as signal injection electrodes on the pipe bottom. AC signal injected used to determine liquid level. The theoretical analysis and experiment results indicate that it is feasible for partially filled pipes.
\end{abstract}

Keywords- Arc-electrode; liquid level; flow velocity; STM32; sensitivity

\section{INTRODUCTION}

As we know, electromagnetic flow meter (EMFM) can measure volume flow ${ }^{[1]-[3]}$, but less effected on fluid density, viscosity temperature and pressure ${ }^{[4][5]}$. The EMFM always is used in the corrosive fluid measurement. It is used in industry widely ${ }^{[6]-[9]}$.

Howerer, most EMFM only used in the full pipe measurement. For fluid under fill or transfer from full pipe to under fill, section area of flow in the pipe is not the cross section area of pipe. Traditional EMFMs cannot get accurate results. The new sensor presented can measure liquid level and flow velocity accurately.

\section{THEORY OF EMFM}

EMFMs are becoming increasingly widely used in water industry and other industries which handle electrically conducting liquids. Figure 1 illustrates the main features of the flow meter. As the liquid passes through magnetic fields, voltage is generated across the pipe and this voltage is measured on a pair of electrodes ${ }^{[10]}$ 。

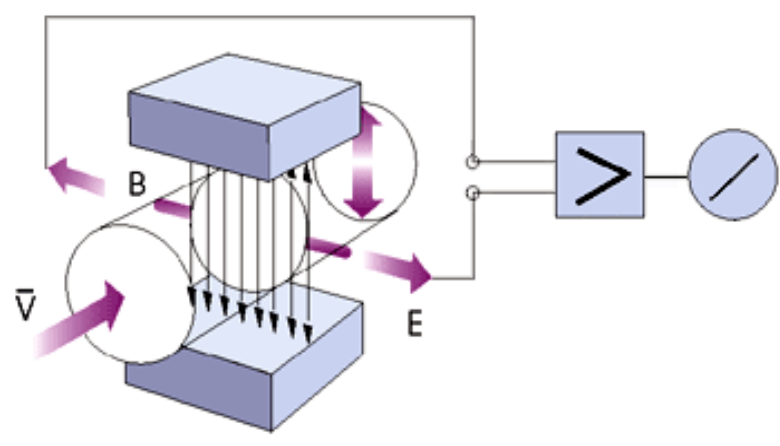

Figure 1. Scheme of EMFM

The relationship of induced electromotive force of electrodes $E$ and velocity (average velocity) ${ }^{\bar{V}}$ is (1) ${ }^{[11]}$ :

$$
E=K B D \bar{v}
$$

And, $B$ is the magnetic flux density;

$D$ is equivalent diameter of measurement pipe;

$K$ is sensor coefficient.

For traditional EMFM, $\bar{v}$ is the average velocity of fluid of full pipe. And $s$ is the section area of measurement pipe, and it is a constant. But as the measurement velocity of partially filled pipes, section area $\boldsymbol{s}$ and liquid level $H$ are must be measured besides $\overline{\boldsymbol{v}}$. The expression is (2),

$$
\boldsymbol{Q}=\overline{\boldsymbol{v}} \bullet \boldsymbol{s}=\frac{\pi \boldsymbol{D}^{2} \boldsymbol{v}}{4}
$$

$Q$ is practical volume flow.

So, traditional EMFM cannot used to measure the velocity in partially filled condition and even cannot detection it.

Compared with traditional single-excitation EMFM, dual-excited EMFM can measure other parameters.

From (1) and (2), it can get (3)

$$
Q=\frac{\pi D E}{4 K B}=A \bullet \frac{E}{B}
$$


$A$ is a constant. For (3), it is a linear relationship between flow $Q$ and induced electromotive force $E$ when diameter $D$ and magnetic induction intensity are constant. So it can get flow through induction potential.

\section{PARTIALly Filled PiPes EMFM}

When pipeline EMFM in working, the pipe under filled may happens.

Or when it flows, the fluid in the pipe turns form full to unfilled. The condition called unfilled pipe EMFM.

In traditional pipe EMFM, it may occurs that the fluid over centerline of pipe but not filled or not over centerline.

Under the theory of traditional EMFM measurement, the section area $S$ or liquid level $H$ must be measured besides average velocity $\overline{\boldsymbol{v}}$.

And the formula of volume flow calculation is (4),

$$
\begin{aligned}
& \boldsymbol{Q}=\boldsymbol{A} \bullet \overline{\boldsymbol{v}} \\
& =\left(\frac{\pi \boldsymbol{D}^{2}}{4}-\frac{\boldsymbol{D}^{2}}{4} \arccos \frac{2 \boldsymbol{h}-\boldsymbol{D}}{\boldsymbol{D}}+\frac{2 \boldsymbol{h}-\boldsymbol{D}}{\sqrt{\boldsymbol{D}^{2}-(2 \boldsymbol{h}-\boldsymbol{D})^{2}}}\right) \cdot \overline{\boldsymbol{v}}
\end{aligned}
$$

$h$ is the depth of liquid level.

\section{A. Theory of multi-parameters in Partially Filled Pipes EMFM}

The pipe for measurement has a pair of arc-electrodes as electrodes used to measure liquid level and velocity.

The arc-electrodes at the bottom of EMFM imposed voltage source signal used for measuring liquid level. When using unfilled-EMFM for measuring liquid level, close the excitation incentive, magnetic field in pipe and set $B=0$, Put AC signals of voltage amplitude constant on excited electrodes.

Liquid coupled in the pipe, voltage signals from measurement electrodes impact liquid level changes. The voltage signals are corresponding with liquid level. The liquid level of the pipe can be got after these signals are dealt with by PC.

In the unfilled EMFM, in spite of the section area is over flow, particles in the flow will induce potential. Theses induced potential must in range of electrodes convergence.

Obviously, electrodes cannot leave flow, or electrodes will not get induced potential. Base on the theory of arcelectrode EMFM, the structure diagrams are shown in Fig2.

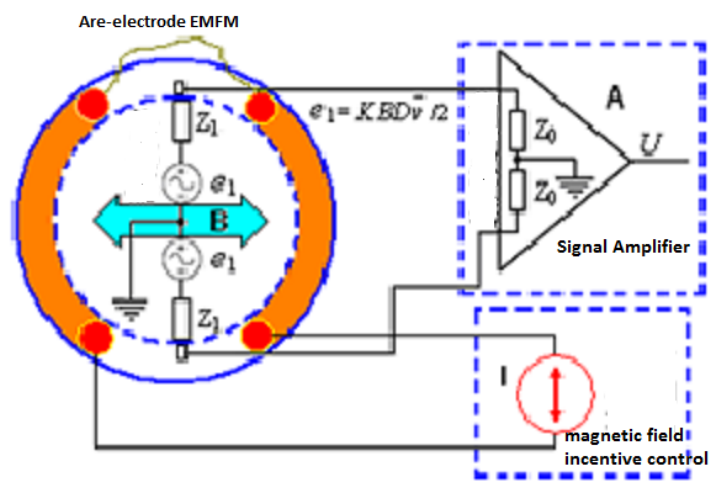

Figure 2. Structure of arc-electrode EMFM
If the location of electrodes of EMFM were put down, and the height is $1 / 10$ of $\mathrm{D}$ (the diameter of the pipe). The EMFM can measure the flow except that below the height.

While as the liquid level over $1 / 10 \mathrm{D}$, the measurement is in high precision (Liquid level always over1/10D). Using excitation- incentive to get velocity, voltage-source-incentive to get liquid level. This is what we called dual-excited theory.

Because the space time of liquid level measuring period and flow velocity measuring period are very short, and it is very shorter than the time of liquid level change, the measurement of liquid level is synchronize with the measurement of the velocity.

\section{B. Hardware Design}

A STM32F103VC is designed as MCU, it has 8 bit DA output and output voltage pulse with $2000 \mathrm{~Hz}$ as excitation signals. The excitation signals amplified by voltage-excited circuit and voltage follower (used to buffer, isolation and improve load-bearing ability). Then the signals into the pair of excited-electrode at bottom of senor. Signals of V0,V2 will be produced by arc-electrodes. And the signals measure velocity and liquid level at the same time.

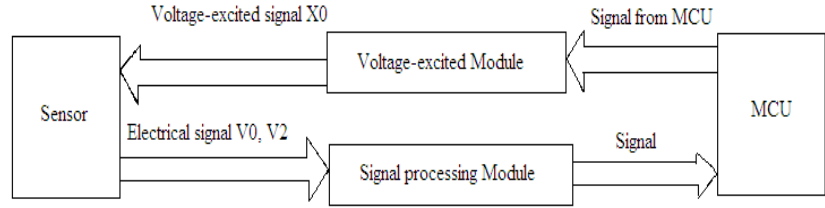

Figure 3. Hardware system structure

A STM32F103 chip is the core of digital signal processing module controls operation of system. Magneticexcited module produces low-frequency rectangular wave. A $120 \mathrm{~mA}$ fixed-current is output to excited-coils. The preceding produces magnetic field to measure flow velocity. Voltage-excited module produces high-frequency rectangular wave of $2000 \mathrm{HZ}$ at the electrodes at the sensor to measure liquid level. Analog signal collection treatment module can collect voltage signals of flow velocity and liquid level. The module also is the unit of denoising and amplifying and filtering.

Partly of signal processing schematic diagram as Fig4.

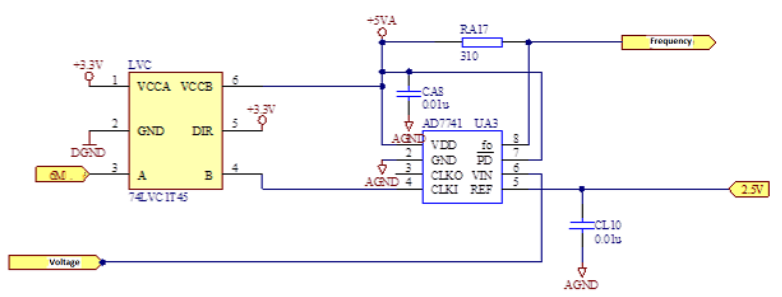

Figure 4. Signal Processing Schematic Diagram

\section{Software Design}

The uC/OS-II is used in program system. It is a source code that has lots of characters like a kind of open source code, portable. It has the character of modern operating 
system and could be used by 8 bit or 32 bit MCU, ARM or DSP. And it has others characters like:

(1)Users compile the function as they need, and need not compiles others.

(2)Execution time is certainty.

(3)Every task has its own stack. And it has coordination ability for the tasks running.

(4)Interrupt hang on running task.

So it is stability and reliability.

A part of program like this:

char value;

char filter()

\{

char new_value;

new_value = get_ad () ;

if ( ( new_value - value > A ) $\|($ value - new_value $>$ A ) return value;

return new_value;

\}

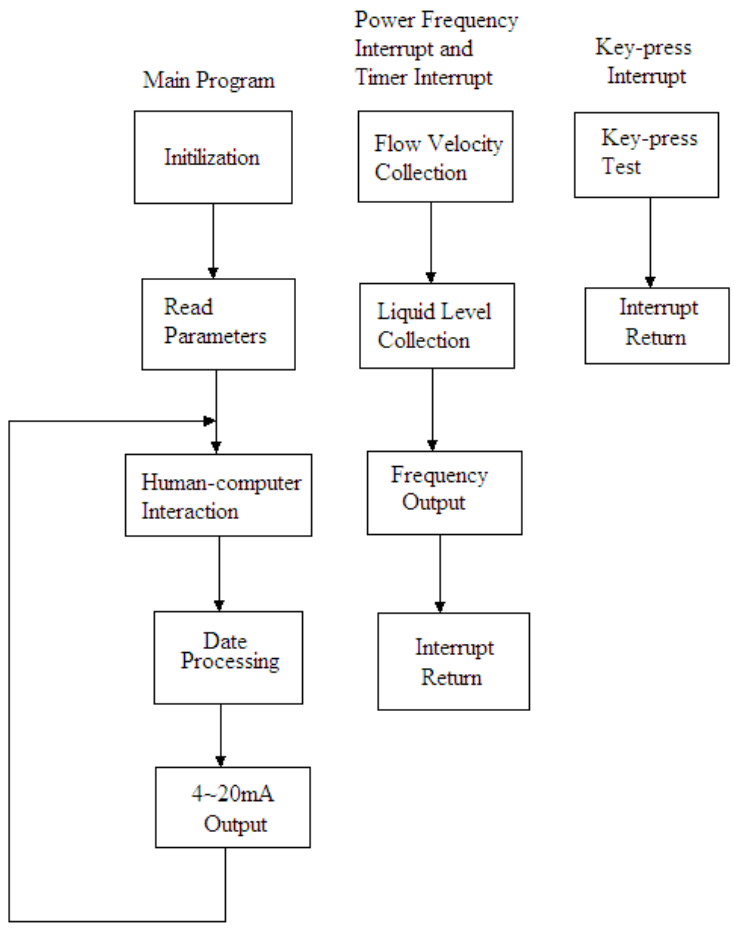

Figure 5. Software system Process

Experiment results and Analysis

The EMFM measures liquid level signals through electric potential signal of electrodes.

Experimental device use PVC pipe as measuring pipe. At mean time PEFF is used as lining too.

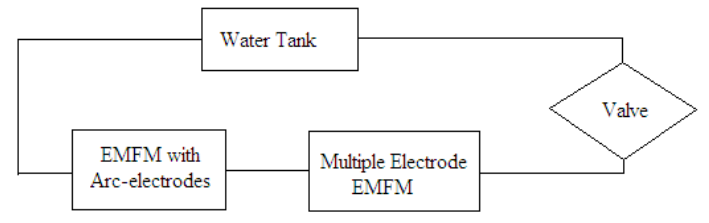

Figure 6. Experiment system structure

$$
k=\frac{\frac{|V o-V i|}{V i}}{\frac{|h o-h i|}{D}}
$$

$k$ is sensor liquid level measurement sensitivity

$V i$ is electric-excited input, $D$ is the diameter of the pipe, Liquid level changes from hi to ho.

Electrode measuring signal changes from $\mathrm{Vi}$ to $\mathrm{Vo}$.

Comparing arc-electrodes EMFM with multi-electrodes EMFM and the results are shown in table I.

From the result, under the condition of full-filled, liquid level changes from 0.6 to 0.9 , liquid -level measurement sensitivity of arc-electrodes EMFM is higher than multielectrodes EMFM’s.

TABLE I. EXPEERMENT RESULTS

\begin{tabular}{|c|c|c|}
\hline $\begin{array}{c}\text { liquid } \\
\text { level }\end{array}$ & $\begin{array}{c}\text { Sensitivity of multi- } \\
\text { electrodes EMFM }\end{array}$ & $\begin{array}{c}\text { Sensitivity of arc- } \\
\text { electrodes EMFM }\end{array}$ \\
\hline 0.1 & 7.1 & 6.9 \\
\hline 0.2 & 1.38 & 1.10 \\
\hline 0.3 & 1.13 & 0.96 \\
\hline 0.4 & 0.75 & 0.69 \\
\hline 0.5 & 0.42 & 0.37 \\
\hline 0.6 & 0.36 & 0.46 \\
\hline 0.7 & 0.21 & 0.33 \\
\hline 0.8 & 0.11 & 0.30 \\
\hline 0.9 & 0.05 & 0.24 \\
\hline 1.0 & 0.02 & 0.06 \\
\hline
\end{tabular}

IV. RESUlt

Analysis and experiment data show that, arc-electrodes EMFM measure liquid level of unfilled pipe EMFM is viable. Sensor has high measurement sensitivity on liquid level and the characteristic of use less external-connection cable. The are-electrodes EMFM is used to measure liquid level and flow velocity of under unfilled condition.

\section{ACKNOWLEDGMENT}

The project is supported by the Solar Photovoltaic Engineering Center of Suzhou Vocational University, and we also thank the supporting of Jiangsu Province.

\section{REFERENCES}

[1] John Arthur Shercliff,"Electromagnetic flow Measurement”, Cambridge University Press, England, 1987.

[2] Mark Bevir, "The theory of induced voltage electromagnetic flowmeters”, Journal of Fluid Mechanics, vol. 43, no. 2, pp.577-90, 1970. 
[3] Li Xuejing,Li, Bin, Yin Shiyi, Jiang Yulin, Gao Song;Theoretical Study of Distribution of Induced Current Density for a Novel non Fixed Electrode Electromagnetic Flow Meter, International Journal of Digital Content Technology and its Applications ,2012,6(5),420-426

[4] Li Xuejing, Li Bin;Virtual Current Density as a Means to Verification of Weight Function of Electromagnetic Flow Meter with Noninsulation Surface Pipe, International Journal of Advancements in Computing Technology ,2012,4(17),236-243

[5] Richard W.Miller. Flow Measurement Engineering Handbook[M], McGraw-Hill,2000,11

[6] ABB. Electromagnetic Flowmeter for Full and Partially Full Pipelines, 2001
[7] John Flood. Single-Sensor Measurement of Flow in Filled or Partially Filled Process Pipes. Krohne America, Inc.

[8] John Flood. Single-Sensor Measurement of Flow in Filled or Partially Filled Pipes[J]. Sensors Magazine online,1997

[9] ARM Limited., ARM v7-M Architecture Application Level Reference Manual, 2008

[10] ZHANGX2.ThevirtualeurrentofanelectromagnetieflowmeterinPartiall yfilledPIPes[J]. MeasurementScienee\&Teehnology,1998,9(11),18525

[11] Research on the Electromagnetic Flowmeter for Partially Filled Pipes[D].2012,24-26 